\title{
Recovery and relapse from severe acute malnutrition after treatment: a prospective, observational cohort trial in Pakistan
}

\author{
Nancy M Dale 1,2 , Laila Salim ${ }^{3}$, Lindsey Lenters ${ }^{4}$, Salim Sadruddin ${ }^{5}$, Mark Myatt ${ }^{6}$ and \\ Stanley H Zlotkin $2,7,8, *$ \\ ${ }^{1}$ Tampere Centre for Child Health Research, University of Tampere and Tampere University Hospital, Tampere, \\ Finland: ${ }^{2}$ Centre for Global Child Health, Hospital for Sick Children, 525 University Avenue, Suite 701, Toronto, \\ Ontario, Canada, M5G 2L3: ${ }^{3}$ Save the Children Canada, Toronto, Canada: ${ }^{4}$ Public Health Ontario, Toronto, \\ Canada: ${ }^{5}$ Global Malaria Programme, World Health Organization, Geneva, Switzerland: ${ }^{6}$ Brixton Health, \\ Llawryglyn, UK: ${ }^{7}$ Department of Paediatrics and Department of Nutritional Sciences, Faculty of Medicine, University \\ of Toronto, 555 University Avenue, Toronto, Ontario, Canada, M5G 2X9: ${ }^{8}$ Dalla Lana School of Public Health, \\ University of Toronto, 155 College Street, Toronto, Ontario, Canada, M5T 3M7
}

Submitted 26 June 2017: Final revision received 21 February 2018: Accepted 2 March 2018: First published online 4 April 2018

\begin{abstract}
Objective: Millions of children suffer from severe acute malnutrition (SAM) in low- and middle- income countries. Much is known about the effectiveness of community treatment programmes (CMAM) but little is known about postdischarge outcomes after successful treatment. The present study aimed to evaluate post-discharge outcomes of children cured of SAM.

Design: Prospective, observational cohort study. Children with SAM who were discharged as cured were followed monthly for 6 months or until they experienced relapse to SAM. 'Cure' was defined as a child achieving a midupper arm circumference (MUAC) of $\geq 115 \mathrm{~mm}$ with $\geq 15 \%$ weight gain after loss of oedema. Relapse was defined as a child with MUAC $<115 \mathrm{~mm}$ and/or oedema at any monthly visit.

Setting: Save the Children CMAM programme in Swabi, Pakistan, from January 2012 to December 2014.

Subjects: Children aged 6-59 months ( $n$ 117) discharged as cured from the CMAM programme were eligible for the study and followed for 6 months.

Results: One hundred children (92.6\%) remained free of SAM, eight $(7.4 \%)$ relapsed to SAM, nine (8.3\%) were lost to follow-up and none died. Most relapses occurred within 3 months of discharge (mean time to relapse $73 \cdot 4$ (SD 36.2) d). At enrolment, $90 \%$ had moderate acute malnutrition (MAM) and 10\% were not malnourished. By the end of 6 months, 35\% persisted with MAM and the remaining were not malnourished.

Conclusions: In rural Pakistan, fewer than $10 \%$ of children cured of SAM relapsed.

The first 3 months is the most vulnerable time.
\end{abstract}

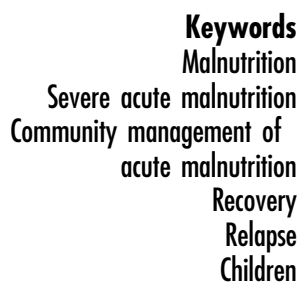

It is estimated that approximately 16 million children under 5 years of age suffer from severe acute malnutrition (SAM) at any given time throughout the world ${ }^{(1)}$. This estimate is based on results of prevalence surveys. When adjusted to account for incidence, this translates to approximately 110 million cases of SAM occurring each year ${ }^{(2)}$. The most common approach for treating SAM is the Community-based Management of Acute Malnutrition (CMAM) model. This model includes short-stay inpatient care in stabilization centres and/or outpatient care in outpatient therapeutic programmes that is often delivered at primary health-care centres. The need for an inpatient phase depends on the presence or absence of medical complications such as anorexia, bilateral pitting oedema, lower respiratory tract infection, high fever, severe dehydration, severe anaemia or dulled sensorium ${ }^{(3-5)}$.

There is considerable literature regarding the effectiveness of CMAM programmes for treating $\mathrm{SAM}^{(6-12)}$. Less work has been done on what happens to these children after they have been discharged as cured. Some work on post-discharge outcomes of children with SAM from inpatient care has been undertaken ${ }^{(13)}$ and summarized ${ }^{(14)}$ and research has described outcomes of children discharged from outpatient care, although the studies are difficult to compare since discharge criteria and lengths of follow-up varied ${ }^{(15-18)}$. Some factors that were identified 
as associated with relapse during 1-year follow-up included mid-upper arm circumference (MUAC) at discharge below $125 \mathrm{~mm}$, no oil/fat consumption during the past $24 \mathrm{~h}$ and incomplete vaccination ${ }^{(18)}$.

There are internationally agreed upon standards for programme effectiveness and coverage of CMAM programmes $^{(19)}$. There are, however, no such standards for post-discharge relapse and mortality. The absence of useful standards reflects, among many things (such as political will, budgetary issues and lack of awareness of the importance of post-discharge care), the absence of evidence that could be used to formulate such standards. The lack of research on the follow-up of patients discharged as cured from CMAM programmes has been identified as a gap in the literature and a research priority ${ }^{(6,7,20-22)}$.

The CMAM model has been endorsed by the WHO, UNICEF and the World Food Programme ${ }^{(23)}$, most international non-governmental organizations, most ministries of health and all donors. CMAM includes a component of active case-finding and community mobilization, but most programmes do little work on post-discharge follow-up other than to report the number of readmissions of previous patients who have been discharged as cured or had defaulted from care prior to formal discharge. In 2012, Save the Children conducted a household survey in Khyber Pakhtunkhwa Province (KPK) of Pakistan and found that $4 \%$ of children aged 6-59 months had SAM (MUAC $<115 \mathrm{~mm}$ ). According to the 2011 National Nutrition Survey in Pakistan, prevalence of SAM had been increasing in $\mathrm{KPK}^{(24)}$. The survey also found that although KPK has relatively low levels of food insecurity compared with other provinces $(68.5 \%$ of the sample reported affordability and accessibility of a variety of foods in all seasons compared with a national average of $42.0 \%$ ), the diets of only $3 \%$ of children aged 6-23 months met the minimum dietary diversity standard (receiving foods from four or more food groups) and only $52 \%$ met the minimum meal frequency standards ${ }^{(24)}$. At this time, Save the Children was rolling out its CMAM programme under the Improving Community Health project in Swabi District of KPK, Pakistan. This programme treated children for SAM. No treatment for moderate acute malnutrition (MAM) was offered. There was also no treatment programme for the children discharged as cured from SAM treatment, as is often found in CMAM programmes.

The aim of the present study was to evaluate the postdischarge outcomes of children aged 6-59 months cured of SAM, up to 6 months after discharge from the CMAM programme run by Save the Children in Swabi District of KPK, Pakistan.

\section{Methods}

The study design was a prospective, observational cohort study conducted by Save the Children Canada and the
Hospital for Sick Children in collaboration with Save the Children Pakistan and Save the Children US.

The study enrolled all eligible children who had been discharged as cured from the outpatient therapeutic programme component of the CMAM programme in Swabi, Topi and Razzar tebsils of Swabi District, Pakistan. Enrolment into the study was ongoing from 1 December 2013 to 29 July 2014. The final follow-up visits for the study were completed in December 2014.

Given that there were no data available on relapse rates in Pakistan and the wide range of relapse rates in the literature, no a priori sample size calculations were done. The sample size was limited to the number of children discharged as cured during the study period.

The CMAM programme was implemented by Save the Children Canada as part of a 3-year collaboration between the Lady Health Workers Program, Department of Health, KPK, Pakistan, Global Affairs Canada and Save the Children, Canada and Pakistan. Lady health workers (LHW) routinely provide preventive care to children and mothers, family planning services, and basic treatment for common illnesses at the village level. Under this collaborative project, community management of SAM was introduced in Pakistan for the first time. Prior to this, the LHW screened children under 5 of years of age for malnutrition and children with SAM were referred to first-level care facilities for further assessment and treatment. In this collaborative project, children identified as having uncomplicated SAM (MUAC $<115 \mathrm{~mm}$ ) who passed the appetite test ${ }^{(20)}$ and were free of medical complications were referred to the outpatient therapeutic programme run by Save the Children Pakistan and treated using the WHO protocol for CMAM programmes ${ }^{(20)}$. The appetite test was performed during the home visits by the LHW instructing the mother on how to give the ready-to-eat therapeutic food (RUTF) to the child and assessing whether the child was able to eat an adequate amount of the sachet per weight ${ }^{(25)}$. Children with bilateral pitting oedema alone or MUAC $<115 \mathrm{~mm}$ and the presence of any grade of oedema, as well as those who did not pass the appetite test, had medical complications or whose level of consciousness was diminished, were referred to the inpatient nutrition programme at the district hospital and were eligible for outpatient treatment once complications had resolved. The CMAM programme only treated children with SAM and the discharge criteria were achieving a MUAC $\geq 115 \mathrm{~mm}$ and $\geq 15 \%$ weight gain and in the absence of oedema. This followed the WHO guidelines at that time, which recommended the use of percentage weight gain for discharge ${ }^{(26)}$. Children were given no further nutritional support after discharge.

The participants for the present study were identified and recruited by the LHW. When it was determined that a child had achieved the discharge criteria (i.e. had been discharged as cured) from the CMAM programme, he/she was enrolled into the study. Inclusion criteria were 
children between 6 and 59 months of age who were discharged as cured from the CMAM programme. Children with congenital anomalies that interfered with their ability to feed by mouth were excluded from the study. Relapse was defined as child with a MUAC $<115 \mathrm{~mm}$ and/or oedema at any of the monthly follow-up visits. If the child was identified as having relapsed, he/she was readmitted to the CMAM programme by the LHW.

At enrolment, the LHW obtained informed consent from the caregivers and baseline data were collected. This included a questionnaire to obtain information on demographic and household-level characteristics, household food insecurity based on the Household Food Insecurity Access Scale (HFIAS) ${ }^{(27)}$, feeding practices and dietary diversity for the child based on Household Dietary Diversity Score (HDDS) ${ }^{(28)}$. Morbidity data as well as height, weight and MUAC measurements were obtained and the absence of oedema confirmed.

The children were followed monthly by the LHW in their respective catchment areas for a 6 -month period or until they experienced relapse to SAM, withdrew from the study or were otherwise lost to follow-up. The caregivers were told to return to the health facility if the child's condition worsened. Field programme assistants provided oversight and supervision throughout the study, including providing relapse verification visits. The monthly followup data included anthropometric measurements (height, weight and MUAC) and oedema, and a questionnaire that included questions on household characteristics, child feeding practices, and any health or dietary-related issues, fever, diarrhoea or vomiting.

\section{Data collection and analysis}

The field team consisted of: (i) a study coordinator responsible for overseeing the implementation of the study and for liaising with the Hospital for Sick Children and Save the Children Pakistan; (ii) a field supervisor, responsible for ensuring data quality and supervision of the field programme assistants; and (iii) field programme assistants, responsible for timely and accurate data collection and support and supervision of the LHW, who in turn were responsible for measurements and administration of questionnaires. The field team was trained on the study design and procedures for data collection, including review of anthropometric measurement techniques, identification of oedema and research ethics standards. For each child, at each data collection point, LHW repeated the anthropometric measurements (height, weight, MUAC) three times following the Intergrowth-21st standards ${ }^{(29)}$. Measurements were taken to the nearest completed unit (i.e. rounded down rather than being rounded up to the nearest unit); MUAC to the nearest $0.1 \mathrm{~cm}$, weight to the nearest $0 \cdot 1 \mathrm{~kg}$, height/length to the nearest $0.1 \mathrm{~cm}$ and age to the nearest month. At the time of data analysis, the mean of three readings of each anthropometric measurement was used.

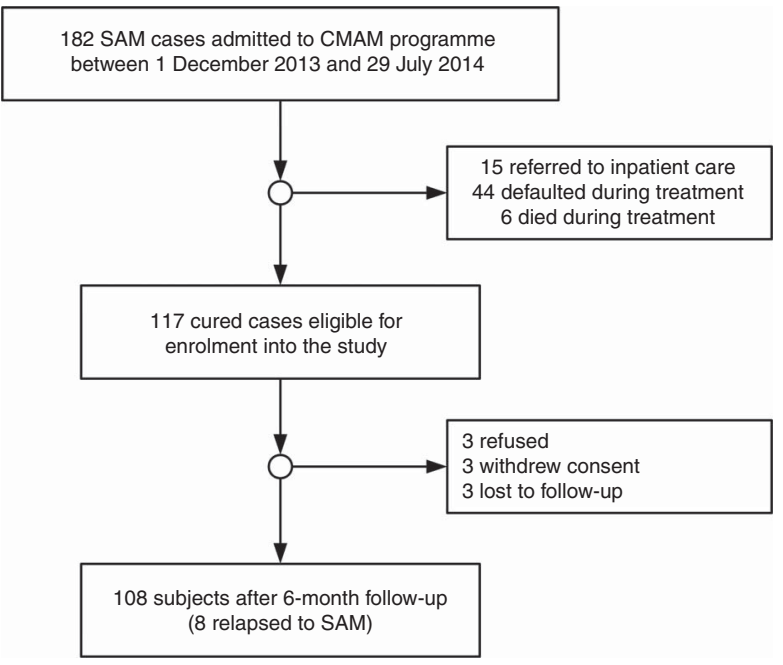

Fig. 1 Study flow diagram of children aged 6-59 months with severe acute malnutrition (SAM) in the Save the Children community-based management of acute malnutrition (CMAM) programme in Swabi District of Khyber Pakhtunkhwa Province, Pakistan; those with SAM who were discharged as cured were followed monthly for 6 months or until they experienced relapse to SAM

Data were captured on paper forms by the LHW during the monthly household visits. Completed paper forms were delivered to the field supervisor who reviewed the forms for completeness. Study data were entered and managed using REDCap (Research Electronic Data Capture) tools hosted at the Hospital for Sick Children. REDCap is a secure, web-based application designed to support data capture for research studies ${ }^{(30)}$. All data entry occurred in Pakistan.

Children were excluded from the study for the following reasons: (i) lost to follow-up (i.e. moved away); (ii) consent withdrawn; and (iii) refusal. There were 182 children admitted into the CMAM programme and 117 children were eligible for enrolment into the study (Fig. 1).

The relapse rate was calculated as a percentage of children who relapsed to SAM of the 108 children followed in the study.

Statistical analyses were performed using the statistical software package SAS versions 9.3 and 9.4, and graphs were produced using R Language and Environment for Statistical Computing version 3.21 software $^{(31)}$. Where applicable, means and proportions were compared between relapse and non-relapse cases using $t$ tests and Fisher's exact test, respectively. A Kaplan-Meier survival curve was used to illustrate time to relapse among children who met the relapse criterion (MUAC $<115 \mathrm{~mm}$ ) during follow-up.

\section{Results}

The mean age of the children was 20 (SD 10) months and $42 \%$ were male. Table 1 describes the children, caregivers 
Table 1 Characteristics of participants in the relapse and nonrelapse groups of children ( $n$ 108) aged $6-59$ months discharged as cured of severe acute malnutrition from the Save the Children community-based management of acute malnutrition programme in Swabi District of Khyber Pakhtunkhwa Province, Pakistan, January 2012-December 2014

\begin{tabular}{lcc}
\hline Characteristic & Non-relapse $(n$ 100) & Relapse $(n 8)$ \\
\hline Child age (months) & 20 & 17 \\
Male sex & 46 & 2 \\
$\quad n$ & 46 & 25 \\
$\%$ & 31 & 25 \\
Age of caregiver (years) & 50 & 4 \\
Child still breast-fed & 50 & 50 \\
$\quad n$ & 7 & 9 \\
$\%$ & 4 & 8 \\
HFIAS† score baseline & & \\
HFIAS† score endline & & \\
\hline
\end{tabular}

*Not including nine subjects who refused to enter the study, withdrew or were lost to follow-up.

†Household Food Insecurity Access Scale: $0=$ no food insecurity; $27=$ severe food insecurity.

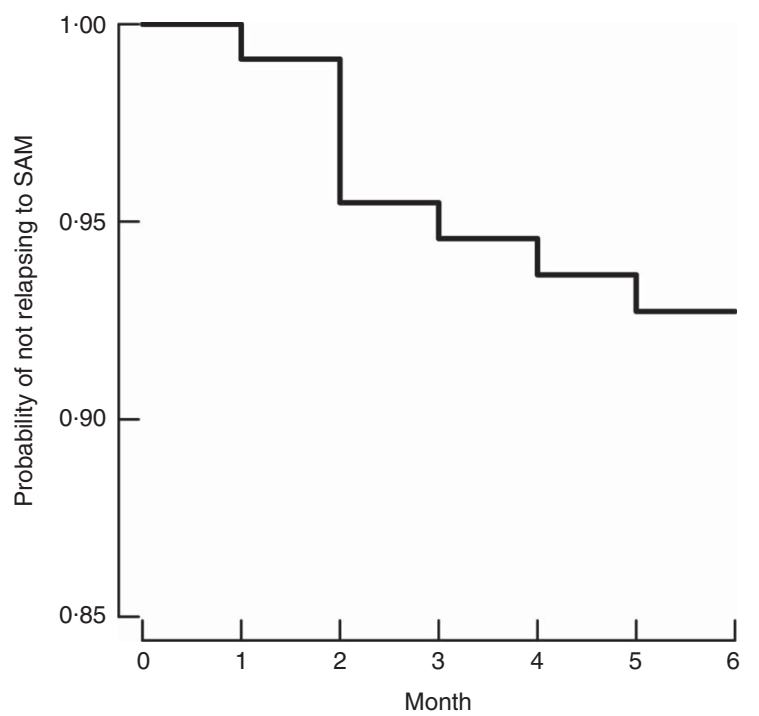

Fig. 2 Kaplan-Meier survival curve for the probability of not relapsing to severe acute malnutrition (SAM) during 6-month follow-up among children ( $n$ 108) aged 6-59 months discharged as cured of SAM from the Save the Children community-based management of acute malnutrition programme in Swabi District of Khyber Pakhtunkhwa Province, Pakistan, January 2012-December 2014

and their households. Eight of 108 cases $(7 \cdot 3 \%$, 95\% CI $3 \cdot 3,14 \cdot 1 \%$ ) relapsed during the 6 months of follow-up with a mean time to relapse of 73.4 (SD 36.2) d. Mean MUAC values differed by $2 \mathrm{~mm}$ at baseline but by $21 \mathrm{~mm}$ at endline. Six out of the eight children who experienced relapse did so within the first 3 months of follow-up, as can be seen in the Kaplan-Meier survival curve (Fig. 2). No deaths occurred during the follow-up period. Figure 3 shows the proportions of children having SAM (MUAC $<115 \mathrm{~mm}$ or oedema), MAM (MUAC 115-124 mm) or no acute malnutrition (MUAC $\geq 125 \mathrm{~mm}$ ) at each month of follow-up. There was a progression from MAM to no

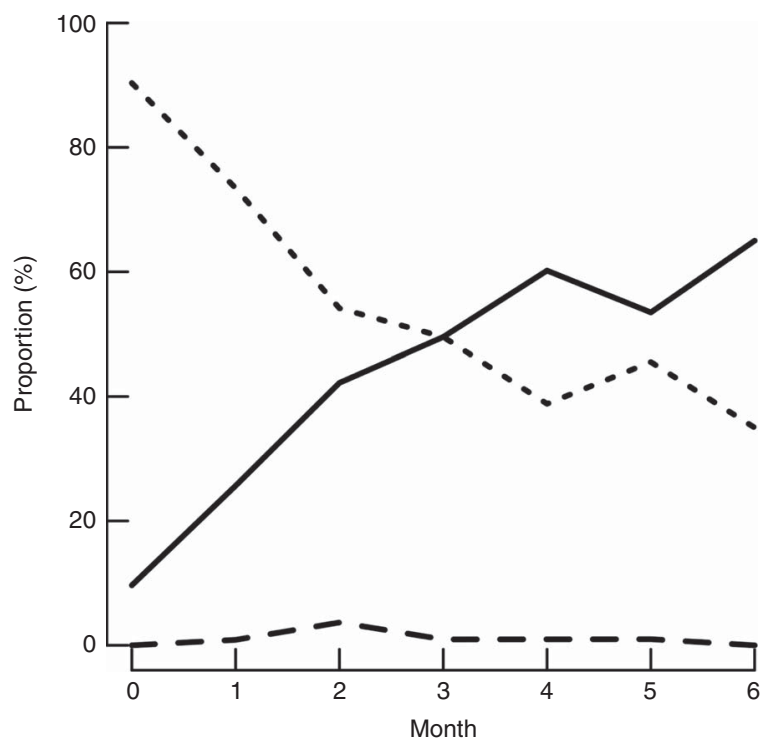

Fig. 3 Proportion of each nutritional status class (- not malnourished; -..-, moderate acute malnutrition; ---, relapsed to severe acute malnutrition (SAM)) during 6-month follow-up among children ( $n$ 108) aged 6-59 months discharged as cured of SAM from the Save the Children community-based management of acute malnutrition programme in Swabi District of Khyber Pakhtunkhwa Province, Pakistan, January 2012-December 2014

acute malnutrition throughout the follow-up period. At the start of the study, $90 \%$ had MAM and $10 \%$ did not have malnutrition. By the end of 6 months, $65 \%$ of children showed no malnutrition while $35 \%$ had MAM. Relapse was a relatively rare event. Although there were differences in the characteristics of those who relapsed, the sample size was too small to perform stratified or disaggregated analyses in general or per age group. Diet diversity and child feeding practices at baseline and endline are reported in the online supplementary material, Supplemental Table 1 . The sample size (the number of relapses) is too small for the analysis to have enough power to be meaningful.

\section{Discussion}

The present study addressed recovery and relapse of previously malnourished children (with SAM) after discharge from a CMAM programme. Of the children, $7 \cdot 3 \%$ relapsed within a 6 -month period of follow-up while more than $50 \%$ transitioned from a moderate malnutrition state (MAM) to 'not malnourished' while receiving no supplementary nutritional interventions after discharge from the treatment programme.

Other authors have examined relapse rates after treatment for SAM. A follow-up study in Bangladesh in 2012 of SAM children (admission based on weight-for-age $Z$-score or oedema; discharge based on attaining weight-for-length of $80 \%$ of the National Center for Health Statistics median) showed a $17 \cdot 8 \%$ relapse rate and $5 \%$ death $^{(15)}$. The admission and discharge criteria for that study are no 
longer used in community programmes and differ from those of other studies, but it does show the vulnerability of children post-discharge from SAM treatment when no supplementary nutritional care is given. The first 3 months showed a high prevalence of illnesses and emphasizes the importance of continued access to health-care facilities for treatment ${ }^{(15)}$. A CMAM programme in Malawi with 3-month post-discharge follow-up found a $1.9 \%$ relapse rate to $\mathrm{SAM}^{(17)}$. However, there were multiple differences from the current study in the treatment and criteria for discharge in the Malawi study. Discharge was based on MUAC $>125 \mathrm{~mm}$, while in the current study discharge was based on MUAC $>115 \mathrm{~mm}$. In Malawi there was an additional 'proof of cure' week before discharge, the provision of a 2-week supply of RUTF at discharge and $29 \%$ of children were enrolled in a supplementary feeding programme at discharge. The children in Malawi had better nutritional status at discharge than the children in the study reported here. An 18-month post-discharge CMAM study in India of children with SAM described relapse rates of $9.1 \%$ at 3 months and $12.0 \%$ at 6 months ${ }^{(16)}$. In that study the discharge criterion was MUAC $\geq 120 \mathrm{~mm}$. The relapse rate of $7.4 \%$ found in the current study is in line with the study from India and, like the Indian study, most relapse cases occurred within the first 3 months (six of eight cases). In the Malawi, India and the study reported here, most relapses occurred soon after discharge.

It is important to note that the Pakistani CMAM programme did not have an associated supplementary feeding programme available for children discharged as cured from the CMAM programme. The degree of household food insecurity, however, was mild in both relapsed and non-relapsed groups (Table 1). All the children were discharged as cured. Most were discharged with MAM and thus still vulnerable to the negative effects of acute malnutrition ${ }^{(32)}$. All children were, however, treated for bacterial and helminthic infections as part of the SAM treatment protocol, received vitamin A, were clinically well and were on a positive growth trajectory. Many emergency programmes continue to treat children until they are cured of MAM or offer some sort of supplementary feeding to support them through the recovery from MAM $^{(32,33)}$. Although we did see recovery from MAM in many of our participants $(65.0 \%, 95 \%$ CI $54.8,74.3 \%)$ after 6 months, the relapse rates in the present study population are likely to be higher than in a study population in which children continued to be treated until complete recovery from both SAM and MAM, as seen in the study of Binns et al. ${ }^{(17)}$. Although ours is not a MAM study, the $35 \%$ who remained with MAM were still vulnerable for further nutritional deterioration and one could argue that a 'bridging ration' or supplementary nutrition programme would be helpful post-discharge to ensure that all children reach a state of adequate nutrition, as emphasized in Ethiopia by James et al. ${ }^{(34)}$. Our study identified that more than half of the relapses occurred within 3 months of discharge from the CMAM programme. Given the small sample size, it was not possible to test for or draw strong conclusions on risk factors, but it seems biologically plausible that children would be most vulnerable to relapse in the first few months after discharge, since this is when many still have MAM and when they are transitioning from a single energy- and micronutrient-dense therapeutic food (RUTF) to a mixed household diet. Research suggests the need to focus on the prevention of associated risk factors beyond the treatment period for SAM, where this is feasible ${ }^{(13)}$, with an emphasis on the first few months following discharge ${ }^{(35)}$. Other useful interventions for reducing relapse in CMAM programmes could be implemented either during the programme period or post-discharge; however, this needs to be further explored ${ }^{(36)}$. Increasing treatment duration by either having a 'proof of cure period' at discharge, where the children need to obtain discharge criteria for two consecutive visits before being eligible for discharge, or by increasing the MUAC discharge threshold are two examples during the programme period ${ }^{(17)}$. Provision of a take-home ration at discharge or discharge to a supplementary feeding programme could be implemented for the post-discharge period. Follow-up of children post-discharge could be done either by community-based workers for the first 3 months or alternatively by the provision of MUAC tapes to mothers, so they can identify relapse on an ongoing basis ${ }^{(37)}$.

These suggested interventions could apply to all programmes that treat and prevent malnutrition. However, they would likely increase total programme costs unless other components of the programme were changed. RUTF is a major programme cost. It may be possible to reduce the RUTF ration once MUAC meets or exceeds $115 \mathrm{~mm}$ to provide similar amounts of nutrients as are provided by MAM treatment programmes ${ }^{(38)}$. It may also be possible to use less expensive ready-to-use supplementary food products, although this may complicate treatment protocols and programme logistics. There may be more efficient and effective approaches than applying a universal intervention to all children to prevent relapse, which is a fairly rare event. Identified risk factors for relapse could be used to target a subset of children at risk of relapsing to SAM; these children could receive extra treatment prior to discharge or receive a take-home ration and/or post-discharge screening. Identification of risk factors for SAM relapse would require further research using considerably larger sample sizes than the study reported here.

The present study has several limitations. First, the sample size was small due to the constraints of the timeline allotted for the study, number of admissions that occurred and proportion of admissions that were discharged as cured during the study period. The sample size allowed the proportion of children relapsing to SAM to be estimated with useful precision. It was, however, too small to allow factors associated with relapse, including dietary 
diversity and feeding practices, to be identified. Relapse is a rare event and a much larger study would be required for such an analysis. Second, it should be noted that the present study investigated relapse observed in children admitted into and discharged as cured from the community-based therapeutic care component of a CMAM programme. Children receiving inpatient care were not recruited into the study. Third, the study lacked a control group which would have allowed us to understand better if relapse rates are worse in post-discharge cured SAM cases compared with the cumulative incidence of SAM in a non-SAM/non-MAM cohort. Another limitation is that the study was done using percentage weight gain as one of the discharge criteria. Since criteria for discharge for MUAC-based programmes no longer recommend the use of percentage weight gain, it would be useful to repeat this study with programmes using MUAC alone, which is the discharge criterion currently recommended ${ }^{(20)}$.

\section{Conclusion}

The present study provides additional information on recovery and post-discharge relapse rates for SAM in CMAM programmes in the context of rural Pakistan. The first 3 months are identified as the most vulnerable time for relapse post-discharge. There is a need for further research to identify risk factors for relapse, particularly in the first 3 months after discharge, and for identifying effective interventions to decrease relapse in a variety of different geographic settings.

\section{Acknowledgements}

Acknowledgements: The authors would like to thank the Ministry of Health in Pakistan, with particular mention of the Lady Health Worker Program Assistant District Coordinator in Swabi, KPK. The authors would also like to thank the many who worked with the Improving Community Health programme and offered valuable input into the programme. Lastly, the authors would like to thank Dr André Briend for his valuable comments on the initial drafts of this manuscript and Nandita Perumal and Ashley Aimone Phillips for their assistance with initial statistical analysis. Financial support: This work was supported by Global Affairs Canada; and Save the Children Canada. Global Affairs Canada had no role in the design, analysis or writing of this article. Staff from Save the Children Canada contributed to the study design, conduct of the study, interpretation of findings and the preparation of the manuscript. Conflict of interest: There were no known financial conflicts of interest. Staff of Save the Children Canada were co-investigators on the study and, as described above, contributed to the study design, conduct of the study, interpretation of findings and the preparation of the manuscript. Authorship: S.H.Z., L.L., L.S. and S.S. conceived of the study and wrote the protocol. N.M.D. and S.H.Z. wrote first drafts of the article. L.L. and N.M.D. completed the data analysis. M.M. assisted with data analysis and presentation and in writing the article. All authors edited the manuscript and approved of the final version. Ethics of buman subject participation: This study was conducted according to the guidelines laid down in the Declaration of Helsinki and all procedures involving human subjects were approved by the Hospital for Sick Children Research Ethics Board (REB\#1000040304) and the Institutional Ethical Review Committee (IERC) of Health Services Academy, Islamabad. Informed consent was obtained from the primary caregivers of each study participant in the form of a signature or thumbprint on the consent form, prior to enrolment into the study. Each caregiver was informed about the details of the study, the right to refuse or withdraw from participation in the study, rights to anonymity and confidentiality, and the absence of any form of payment for participation in the study.

\section{Supplementary material}

To view supplementary material for this article, please visit https://doi.org/10.1017/S1368980018000745

\section{References}

1. UNICEF, World Health Organization \& World Bank Group (2015) Levels and Trends in Child Malnutrition Joint Child Malnutrition Estimates: Key Findings of the 2015 Edition. http://www.who.int/nutrition/publications/ jointchildmalnutrition_2015_estimates/en/ (accessed May 2017).

2. Hure A, Oldmeadow C \& Attia J (2016) Invited commentary: improving estimates of severe acute malnutrition requires more data. Am J Epidemiol 184, 870-872.

3. Collins S (2004) Community-Based Therapeutic Care: A New Paradigm for Selective Feeding in Nutritional Crises. Network Paper no. 48. London: Overseas Development Institute; available at http://www.alnap.org/resource/11334

4. World Health Organization, UNICEF \& United Nations Standing Committee on Nutrition (2006) WHO, UNICEF, and SCN Informal Consultation on Community-Based Management of Severe Malnutrition in Children. SCN Nutrition Policy Paper no. 21. http://www.who.int/nutrition/publications/ severemalnutrition/FNB_0379-5721.pdf (accessed May 2017).

5. World Health Organization, World Food Programme, United Nations System Standing Committee on Nutrition, et al. (2007) Community-Based Management of Severe Acute Malnutrition. http://www.who.int/nutrition/topics/statement_ commbased_malnutrition/en/ (accessed May 2017).

6. Lenters LM, Wazny K, Webb P et al. (2013) Treatment of severe and moderate acute malnutrition in low- and middleincome settings: a systematic review, meta-analysis and Delphi process. BMC Public Health 13, Suppl. 3, S23.

7. Schoonees A, Lombard M, Musekiwa A et al. 2013) Readyto-use therapeutic food for home-based treatment of severe acute malnutrition in children from six months to five years of age. Cochrane Database Syst Rev issue 6, CD009000.

8. Ciliberto MA, Sandige H, Ndekha MJ et al. (2005) Comparison of home-based therapy with ready-to-use therapeutic food with standard therapy in the treatment of malnourished 
Malawian children: a controlled, clinical effectiveness trial. Am J Clin Nutr 81, 864-870.

9. Manary MJ, Ndkeha MJ, Ashorn P et al. (2004) Home based therapy for severe malnutrition with ready-to-use food. Arch Dis Child 89, 557-561.

10. Ndekha MJ, Manary MJ, Ashorn P et al. (2005) Home-based therapy with ready-to-use therapeutic food Is of benefit to malnourished, HIV-infected Malawian children. Acta Paediatr 94, 222-225.

11. Sadler K, Puett C, Mothabbir G et al. (2011) Community Case Management of Severe Acute Malnutrition in Southern Bangladesh. http://www.fic.tufts.edu/assets/CommunityCase-Mgt.pdf (accessed June 2017).

12. Puett C, Sadler K, Alderman H et al. (2013) Costeffectiveness of the community-based management of severe acute malnutrition by community health workers in southern Bangladesh. Health Policy Plan 28, 386-399.

13. Kerac M, Bunn J, Chagaluka G et al. (2014) Follow-up of post-discharge growth and mortality after treatment for severe acute malnutrition (FuSAM Study): a prospective cohort study. PLoS One 9, e96030.

14. Bahwere P, Mtimuni A, Sadler K et al. (2012) Long term mortality after community and facility based treatment of severe acute malnutrition: analysis of data from Bangladesh, Kenya, Malawi and Niger. J Public Health Epidemiol $\mathbf{4}$, 215-225.

15. Ashraf H, Alam N, Chisti MJ et al. (2012) A follow-up experience of 6 months after treatment of children with severe acute malnutrition in Dhaka, Bangladesh. $J$ Trop Pediatr 58, 253-257.

16. Burza S, Mahajan R, Marino E et al. (2016) Seasonal effect and long-term nutritional status following exit from a community-based management of severe acute malnutrition program in Bihar, India. Eur J Clin Nutr $\mathbf{7 0}$ 437-444.

17. Binns P, Dale NM, Banda T et al. (2016) Safety and practicability of using mid-upper arm circumference as a discharge criterion in community based management of severe acute malnutrition in children aged 6 to 59 months programmes. Arch Public Health 74, 24.

18. Somassè YE, Dramaix M, Bahwere P et al. (2016) Relapses from acute malnutrition and related factors in a communitybased management programme in Burkina Faso. Matern Child Nutr 12, 908-917.

19. The Sphere Project (2011) Humanitarian Charter and Minimum Standards in Humanitarian Response: The Sphere Handbook. http://www.sphereproject.org/handbook/ (accessed June 2017)

20. World Health Organization (2013) Guideline: Updates on the Management of Severe Acute Malnutrition in Infants and Children. http://www.apps.who.int/iris/bitstream/10665/95584/ 1/9789241506328_eng.pdf?ua=1 (accessed May 2017).

21. Petri WA, Naylor C \& Haque R (2014) Environmental enteropathy and malnutrition: do we know enough to intervene? BMC Med 12, 187.

22. Picot J, Hartwell D, Harris P et al. (2012) The effectiveness of interventions to treat severe acute malnutrition in young children: a systematic review. Health Technol Assess 16, issue $19,1-316$.

23. World Health Organization, UNICEF, World Food Programme, et al. (2007) Community-Based Management of Severe Acute Malnutrition: A Joint Statement. http://www. who.int/maternal_child_adolescent/documents/a91065/en/ (accessed June 2017).

24. Butta ZA (2011) National Nutrition Survey Pakistan. Karachi: Government of Pakistan.

25. CMAM_Training_Mod4_ENGLISH_Nov2008.pdf (n.d.) Trainer's Guide. Community-Based Management of Acute Malnutrition. Module Four: Outpatient Care for the
Management of SAM Without Medical Complications. http:// www.fhi360.org/sites/default/files/media/documents/CMAM_ Training_Mod4_ENGLISH_Nov2008.pdf (accessed December 2017).

26. World Health Organization \& UNICEF (2009) WHO Child Growth Standards and the Identification of Severe Acute Malnutrition in Infants and Children: A Joint Statement by the World Health Organization and the United Nations Children's Fund. http://www.who.int/nutrition/publications/ severemalnutrition/9789241598163/en/index.html (accessed June 2017).

27. Food and Nutrition Technical Assistance (2007) Household Food Insecurity Access Scale (HFIAS) for Measurement of Food Access: Indicator Guide. http://www.fantaproject.org/ monitoring-and-evaluation/household-food-insecurity-accessscale-hfias (accessed June 2017).

28. Food and Nutrition Technical Assistance (2006) Household Dietary Diversity Score (HDDS) for Measurement of Household Food Access: Indicator Guide VERSION 2. http://www.fantaproject.org/sites/default/files/resources/ HDDS_v2_Sep06_0.pdf (accessed January 2018).

29. The International Fetal and Newborn Growth Consortium (2012) INTERGROWTH-21st International Fetal and Newborn Growth Standards for the 21st Century: Anthropometry Handbook. http://www.intergrowth21.tghn.org/site_media/ media/articles/Anthropometry_Handbook_April_2012.pdf (accessed December 2017).

30. Harris P, Taylor R, Thielke R et al. (2009) Research electronic data capture (REDCap) - a metadata-driven methodology and workflow process for proving translational research informatics support. J Biomed Inform 42, 377-381.

31. R Development Core Team (2016) R: A Language and Environment for Statistical Computing. http://www.Rproject.org/ (accessed June 2017).

32. Annan RA, Webb P \& Brown R (2014) Management of Moderate Acute Malnutrition: Current Knowledge and Practice. CMAM Forum Technical Brief. https://www.ennonline.net/ attachments/2289/MAM-management-CMAM-Forum-TechnicalBrief-Sept-2014.pdf (accessed March 2018).

33. Lenters L, Wazny K \& Bhutta Z (2016) Management of severe and moderate acute malnutrition in children. In Reproductive, Maternal, Newborn, and Child Health: Disease Control Priorities, 3rd ed., vol. 2, pp. 205-224 [RE Black, R Laxminarayan, M Temmerman et al., (editors]. Washington, DC: The International Bank for Reconstruction and Development/The World Bank; available at http://www.ncbi.nlm.nih.gov/books/ NBK361900/.

34. James P, Sadler K, Wondafrash M et al. (2016) Children with moderate acute malnutrition with no access to supplementary feeding programmes experience high rates of deterioration and no improvement: results from a prospective cohort study in rural Ethiopia. PLoS One 11, e0153530.

35. Roy SK, Chowdhury AK \& Rahaman MM (1983) Excess mortality among children discharged from hospital after treatment for diarrhoea in rural Bangladesh. Br Med J (Clin Res Ed) 287, 1097-1099.

36. Stobaugh HC, Bollinger LB, Adams SE et al. (2017) Effect of a package of health and nutrition services on sustained recovery in children after moderate acute malnutrition and factors related to sustaining recovery: a clusterrandomized trial. Am J Clin Nutr 106, 657-666.

37. Alé F, Phelan K, Issa H et al. (2016) Mothers screening for malnutrition by mid-upper arm circumference is non-inferior to community health workers: results from a large-scale pragmatic trial in rural Niger. Arch Public Health 74, 38.

38. Amanda M, Koroma AS, Abla C et al. (2015) Severe and moderate acute malnutrition can be successfully managed with an integrated protocol in Sierra Leone. $J$ Nutr $\mathbf{1 4 5}$, 2604-2609. 